Volume 9, No.5, September - October 2020

International Journal of Advanced Trends in Computer Science and Engineering

Available Online at http://www.warse.org/IJATCSE/static/pdf/file/ijatcse254952020.pdf

https://doi.org/10.30534/ijatcse/2020/254952020

\title{
OLAP-Tools for the Formation of Connected and Diversified Production and Project Management Systems
}

\author{
Petro Kulikov ${ }^{1}$, Galyna Ryzhakova ${ }^{2}$, Tetyana Honcharenko ${ }^{3}$, Dmytro Ryzhakov ${ }^{2}$ and Oksana Malykhina ${ }^{2}$ \\ ${ }^{1}$ Professor, Rector of Kyiv National University of Construction and Architecture, Ukraine \\ ${ }^{2}$ Department of Management in Construction, Kyiv National University of Construction and Architecture, \\ Ukraine, Galyna_Ryzhakova@ukr.net \\ ${ }^{3} \mathrm{PhD}$ (Engineering), Associate Professor, Department of Information Technology, Kyiv National University of \\ Construction and Architecture, Ukraine, iust511@ukr.net
}

\begin{abstract}
This article is devoted to the problem of the ability to represent business processes of complex systems of project and production management as a set of interacting semantically defined objects. To solve this problem, multidimensional modeling based on OLAP technology is proposed. The model covering the information system is presented in the form of a Metabase, which contains information about each type of instance of an independent business process. A mathematical description of the model of the subject area from the standpoint of OLAP-tools is given. It is proposed to use a multidimensional data model as a hypercube, the edges of which are sequences of values of the analyzed parameters for multidimensional analysis of the subject area. A multidimensional database is considered as an object archive designed to store the attributes of passive and active objects. Each OLTP system is an implementation of the subject area model and is mapped to a multidimensional matrix as OLAP-cube. The application of this concept in a Web-based environment is covered.
\end{abstract}

Key words: OLAP, multidimensional model, data space, production and project management systems, Metabase.

\section{INTRODUCTION}

The world around us is multidimensional and each object is characterized by a set of specific parameters. When modeling a real object or process to solve a certain problem, as a rule, it is necessary to reduce the dimension of the world and select the range of significant parameters that are of interest for research. It is often necessary to build dependencies between various parameters, the number of which can vary within wide limits in the process of analyzing data and searching for solutions to a given problem. Currently, the process approach for organizing project management, based on formal models of the life cycle (LC) of systems, is widely used. Many types of products are complex systems, based on the interaction of a set of managerial and technical actions, including technical means, software and the human factor. Their production is carried out using processes, having a variety of technical and control "inputs" and "outputs".

At the same time, the role of standards used in at all stages of management, primarily because the standards provide for the interaction of various components with each other.

It is necessary to define independent business processes as schemas of information objects of the considered model. The rules for interaction between instances of business processes can be evaluated by two types of properties. The first type includes properties that can be quantified. The second type includes qualitative properties, which require pair wise comparison of objects with the property being evaluated in order to determine their place in relation to the concept under consideration.

Authors' works [1-3] analyze basic elements of the innovation-analytical platform and management tools of business processes in construction.

In the works [4-5], the authors provide the reengineering of the business processes in construction based on BIM-technology.

The articles [6-8] give a detailed review of innovative tools for management the lifecycle of strategic objectives of the enterprise-stakeholder in construction.

In the work [9], the authors propose modeling of construction site based on multidimensional information objects.

The articles [10] and [11] are devoted to the structural information management of production systems in construction and construction of models for conversion of mortgage applications by the method of multiple regression and neural networks.

The authors of the works [12-14] formalize a concept of parametric modeling of construction objects.

The authors of [15-17] examine construction of membership functions in fuzzy modeling tasks using the analytic hierarchy process.

Authors' works [18-19] are devoted to the method for evaluation of educational environment subjects' performance based on the calculation of volumes of M-simplexes and fractal time series analysis in non-stationary environment.

This research discusses the problem of the ability to represent business processes of complex systems of project and 
production management as a set of interacting semantically defined objects. To solve this problem, multidimensional modeling based on OLAP technology is proposed.

\section{THE MAIN RESEARCH}

A model covering an information system can be represented in the form of a Metabase, which contains information on each type of accounting object, in our case, about each instance of an independent business process. Let's designate such a model of an information system as the set $\boldsymbol{G}$.

On the other hand, the information system can be represented in the form of a functional system. That is in the form of a set of functions. Let's designate such a model of an information system as the set $\boldsymbol{F}$.

Let's introduce the notation:

$\boldsymbol{Q}_{\boldsymbol{G}}$ is a set of properties determined by the relations between elements of the set $\boldsymbol{G}$.

$\boldsymbol{Q}_{\boldsymbol{F}}$ is a set of properties determined by the relationships between elements of the set $\boldsymbol{F}$.

$\boldsymbol{Q}_{\boldsymbol{F} G}$ is a set of properties determined by connections between elements of the sets $\boldsymbol{F}$ and $\boldsymbol{G}$.

Then the relations within the sets $F$ and $G$ can be respectively determined by relations on the Cartesian product:

$$
Q_{F} \times F=\left\{\begin{array}{l}
z_{i}^{F}=\left(q_{i}^{F}, f_{i}\right): q_{i}^{F} \in Q_{F}, \\
f_{i} \in F, i=1, \ldots, n
\end{array}\right\}
$$

and

$$
Q_{G} \times G=\left\{\begin{array}{l}
z_{i}^{G}=\left(q_{i,}^{G} g_{i}\right): g_{i}^{G} \in Q_{G}, \\
g_{i} \in G, i=1, \ldots, n
\end{array}\right\}
$$

The relation between them can be determined by the relation on the Cartesian product

$$
Q_{F G} \times G \times F=\left\{\begin{array}{l}
z_{i}^{F G}=\left(q_{i}^{F G}, g_{i}, f_{i}\right): q_{i}^{F G} \in Q_{F G}, \\
g_{i} \in G, f_{i} \in F, i=1, \ldots, n
\end{array}\right\}
$$

The belonging of the element $z_{i}^{F G}$ to this relation can be interpreted as follows: "the element $g_{i}$ of the information system contains information on the property $q_{i}^{F G}$ of the functional part of the information system $f_{i}$ ".

Searching for information corresponding to a particular element $f_{i}$ in $g_{i}$ is reduced to determining the ratio $R \subseteq G \times$ $F$. Thus, for any pair $\left(g_{i}, f_{i}\right) \in R: g_{i} \in G, f_{i} \in F, i=1, \ldots, n$, we can say that fi is relevant to $g_{i}$, and the solution to the problem of determining the relevance of elements of the sets $G$ and $F$ is reduced to the definition of the relation $R \subseteq G \times F$. Moreover, $\forall g_{i} \in G, f_{i} \in F, g_{i} \in G, f_{i} \in F, i, j=1, \ldots, n$, it is true that if $f_{i} \subseteq f_{j}$ and $g_{i} \subseteq g_{i}$, that is, all elements $g_{i}$ are contained in $g_{i}$ and all elements $f_{i}$ are contained in $f_{i}$ and $\left(g_{i}\right.$, $\left.f_{i}\right) \in R$, then $\left(g_{i}, f_{i}\right) \in R$. Except in the extreme case, when the relation $R$ is the Cartesian product $G \times F$ itself, the relation does not include all possible tuples from the Cartesian product. This means that for each relation there is a criterion for determining which tuples are included in the relation and which are not. Thus, each relation $R$ can be associated with a logical expression (predicate) $Q_{F G}$, which depends on a certain number of parameters ( $n$-ary predicate) and determines whether the tuple $\left(g_{i}, f_{j}\right)$ belongs to the relation $R$. Thus, the membership of a tuple in the relation is equivalent to the truth of the predicate:

$$
\left(g_{j}, f_{j}\right) \in R \Leftrightarrow\left\{Q_{F G}\right\}=\{G, F, R\}
$$

However, in any case, with the information approach to formalize the subject area, the categories of objects and the relations between them are primary, i.e., formally, the system of relations can be represented by a set of objects in the subject area and a variety of relations between them. The characteristic of the process can be represented as a set of pairs $\left\{\left\langle A_{i}, D_{i}\right\rangle, i=1, \ldots, n\right\}$, where $A_{i}$ is a non-empty set of property names (attributes), $D_{i}$ is a set of values of the corresponding attributes. Values are broken down into object classes that interact with each other based on rules. Let $\pi$ be the set of these rules. Relationships $G=\{\bar{G}, \widetilde{G}\}$ can be established on a set of attributes, which are divided into quantitative $\bar{G}$ and qualitative $\tilde{G}$, for which many types of assessment are defined, for example $T=\{$ "projects are moving towards achieving their goals", "projects are being conducted in accordance with the relevant directives", "projects are being implemented as planned", "projects remain viable" $\}$. Then any estimation rule can be represented by a tuple $\pi=\langle G, T\rangle$.

Thus, the set of information characteristics of the process $\left\{\left\langle A_{i}, D_{i}\right\rangle, i=1, \ldots, n\right\}$, the established relations $G=\{\bar{G}, \widetilde{G}\}$, and the rules for establishing relations $\pi=\langle G, T\rangle$ can be used to formally define the process in the form the following tuple of components:

$$
Z=\left\{\left\langle A_{i}, D_{i}\right\rangle,\{\bar{G}, \tilde{G}\},\langle\{\bar{G}, \tilde{G}\}, T\rangle\right\}, i \in N
$$

Attribute values may not be numeric. In particular, the linguistic form of data presentation is widely used in macroeconomic, sociological, marketing, medical, legal data warehouses. To assess the characteristics that are of a qualitative nature, ordinal scales can be used, the point elements of which correspond to the gradations of the verbal scales. We can assign the values of linguistic variables to the levels of ordinal scales and perform all further operations with their membership functions. At the same time, their adequacy cannot be verified by the means of theory, and each existing method for constructing a membership function formulates its own requirements and justifications for the choice of just such a construction.

Consider $N$ objects for which the intensity of manifestation of characteristics of attributes with the names $A_{j}, j=1, \ldots, k$ is estimated, the values of which $X_{j}, j=1, \ldots, k$ are used to assess the qualitative characteristic $Y$. From the standpoint of the apparatus of the theory of fuzzy sets, the models of expert evaluation of features are full orthogonal semantic spaces [9], where normal triangular numbers and $T$-numbers are used as 
membership functions. This method allows you to go from diverse qualitative information to a single abstract value - the value of the membership function. We use the method of working with fuzzy information, considered in [9].

Let $X_{i j}, i=1, \ldots, m_{j}$ are levels of verbal scales used to assess attributes with the names $A_{j}, j=1, \ldots, k$ and arranged in ascending order of intensity of their manifestation.

Let's denote by $a_{i}^{j}, i=1, \ldots, m_{j}, j=1, \ldots, k$ - the relative numbers of objects referred during the assessment of attributes with the names $A_{j}, j=1, \ldots, k$ to the level $X_{i j}, i=$ $1, \ldots, m_{j}, j=1, \ldots, k, \sum_{i=1}^{m} a_{i}^{j}=1, j=1, \ldots, k$.

We can construct $k$ sets of fuzzy numbers corresponding to attributes named $A_{j}, j=1, \ldots, k$. Let $\mu_{i j}(x)$ denote the membership function of the fuzzy number $\tilde{X}_{i j}$ corresponding to the $i$-th level of the $j$-th attribute $i=1, \ldots, m_{j}, j=1, \ldots, k$. Fuzzy numbers can be taken as an estimate of the object, $\tilde{X}_{i j}$, $i=1, \ldots, m_{j}, j=1, \ldots, k$ with the corresponding membership functions $\mu_{i j}(x), i=1, \ldots, m_{j}, j=1, \ldots, k$. Then the estimate of the $n$-th object by the attribute $X_{j}, j=1, \ldots, k$ can be represented by the membership function $\mu_{j}^{n}$. Using the so-called L-R membership function, the assessment of each object can be written in the form

$$
\begin{gathered}
\left\{\mu_{j}^{n}(x)=\left(a_{j 1}^{n}, a_{j 2}^{n}, a_{j L}^{n}, a_{j R}^{n}\right)\right\}, \\
n=1, \ldots, N, j=1, \ldots, k
\end{gathered}
$$

The specific form of member functions is determined taking into account the specifics of the existing uncertainty, the real situation at the object and the number of degrees of freedom in functional dependence. One of the most convenient for describing the membership function is monotonically increasing or decreasing sigmoid, they are convenient for specifying linguistic terms in a natural language, the equations of which have the form: $\mu(x)=\frac{1}{1+e^{-a(x-b)}}$ for an increasing function and $\mu(\mathrm{x})=\frac{1}{1-\left(1+\mathrm{e}^{-\mathrm{a}(\mathrm{x}-\mathrm{b})}\right)}$ for a decreasing one, where $a$ is the slope of the sigmoid, $b$ - allows you to shift the center point along the axis. At $a=0$, the sigmoid degenerates into a straight line at the 0.5 mark.

It is advisable to designate the weighting coefficients of the estimated attributes by $\omega_{j}, j=1, \ldots, k, \sum_{j=1}^{n} \omega_{j}=1$. The overall fuzzy estimate of the $n$-th object $(n=1, \ldots, N)$ can be determined by the sum of the estimates for all attributes taking into account the weight coefficients $\tilde{A}^{n}=\omega_{1} \otimes \tilde{X}_{11}^{n} \oplus \ldots \oplus \omega_{1}$ $\otimes \tilde{X}_{m_{k} k}^{n}$ and the membership function

$$
\mu_{n}(x)=\left(\begin{array}{c}
\sum_{j=1}^{k} \omega_{j} a_{j 1}^{n}, \Sigma_{j=1}^{k} \omega_{j} a_{j 2}^{n}, \\
\sum_{j=1}^{k} \omega_{j} a_{j L}^{n}, \Sigma_{j=1}^{k} \omega_{j} a_{j R}^{n}
\end{array}\right), n=1, \ldots N .
$$

The fuzzy number $\tilde{B}_{1}$, corresponding to the lowest intensity is determined by $\tilde{B}_{1}=\omega_{1} \otimes \tilde{X}_{11}^{n} \oplus \ldots \oplus \omega_{k} \otimes \tilde{X}_{1 k}^{n}$, and $\tilde{B}_{m}$ corresponding to the highest intensity:

$$
\tilde{B}_{m}=\omega_{1} \otimes \tilde{X}_{m_{1} 1}^{n} \oplus \ldots \oplus \omega_{k} \otimes \tilde{X}_{m_{k} k}
$$

The obtained fuzzy numbers can be defasified, for example, by the method of the center of gravity, the resulting clear numbers will be denoted as $Z_{n}, \mathrm{n}=1, \ldots, \mathrm{N}$, $B_{1}, B_{m}$, respectively. These numbers can be used as a normalized rating assessment of the $\mathrm{n}$-th object, $\mathrm{n}=1, \ldots$, $\mathrm{N}$, according to the formula:

$$
E_{n}=\frac{Z_{n}-B_{1}}{B_{m}-B_{1}}, n=1, \ldots, N
$$

In the general case, the characteristic of each object $X_{i}$ can be described by the corresponding linguistic variable:

$$
X_{i}=<A_{j}, T_{j}, D_{j}>\text {, }
$$

where $T_{j}=\left\{T_{1}^{j}, T_{2}^{j}, \ldots, T_{m_{j}}^{j}\right\}$ is the term set of the linguistic variable $A_{j}$ (a set of linguistic values of the attribute), $m_{j}$ is number of attribute values; $D_{j}$ is (subject scale) base set of the attribute $A_{j}$. To describe the terms $T_{k}^{j}, \mathrm{k}=1, \ldots$, $m_{j}$ corresponding to the values of the attribute $A_{j}$, fuzzy variables $\left\langle T_{k}^{j}, D_{j}, \tilde{C}_{k}^{j}\right\rangle$ can be used, i.e. the value $T_{k}^{j}$ is described by the fuzzy set $\tilde{C}_{k}^{j}$ in the base set $D_{j}$ :

$$
\tilde{c}_{k}^{j}=\left\{\left\langle\mu_{c_{k}^{j}}(d) \mid d\right\rangle\right\}, d \in D_{j}, k=1, \ldots, m_{j}
$$

Then the fuzzy set of the second level can be taken as a fuzzy characteristic of the object $x_{i}$

$$
\begin{gathered}
\tilde{x}_{i}=\left\{\left\langle\mu_{x_{i}}\left(a_{j}\right) \mid a_{j}\right\rangle\right\}, \\
\mu_{x_{i}}\left(a_{j}\right)=\bigcup_{k=1}^{m_{j}}\left\{\left\langle\mu_{\mu_{x_{i}}}\left(T_{k}^{j}\right) \mid T_{k}^{j}\right\rangle\right\}, T_{k}^{j} \in T_{j}, a_{j} \in A_{i}
\end{gathered}
$$

All this makes it possible to move from diverse qualitative information to one abstract quantity - the value of the membership function. To assess the qualitative characteristics of objects, we use the following representation of their elements [7, 9]:

- the membership function for the extreme term-set corresponding to the minimum intensity of the feature manifestation can be represented as

$$
\mu_{x_{1}}(x)=\left\{\begin{array}{l}
1,0 \leq x \leq a_{1}-\frac{1}{2} \min _{a}\left(a_{1}, a_{2}\right) \\
1-\frac{x-\left(a_{1}-\frac{1}{2} \min _{a}\left(a_{1}, a_{2}\right)\right)}{\min _{a}\left(a_{1}, a_{2}\right)} \\
a_{1}-\frac{1}{2} \min _{a}\left(a_{1}, a_{2}\right)<x \leq a_{1}+\frac{1}{2} \min _{a}\left(a_{1}, a_{2}\right) \\
0, a_{1}+\frac{1}{2} \min _{a}\left(a_{1}, a_{2}\right)<x \leq 1
\end{array}\right.
$$

- the membership function for the extreme term-set corresponding to the maximum intensity of the manifestation of the feature can be represented as

$$
\begin{gathered}
\mu_{x_{m}}(x)= \\
\left\{\begin{array}{l}
0,0 \leq x \leq 1-a_{m}-\frac{1}{2} \min _{a}\left(a_{m-1}, a_{m}\right) \\
1+\frac{x-\left(1-a_{m}-\frac{1}{2} \min \left(a_{m-1}, a_{m}\right)\right)}{\min _{a}\left(a_{m-1}, a_{m}\right)} \\
1-a_{m}-\frac{1}{2} \min _{a}\left(a_{m-1}, a_{m}\right)<x \leq 1-a_{m}+\frac{1}{2} \min _{a}\left(a_{m-1}, a_{m}\right) \\
1,1-a_{m}+\frac{1}{2} \min _{a}\left(a_{m-1}, a_{m}\right)<x \leq 1
\end{array}\right.
\end{gathered}
$$


- the membership function for average term sets of a qualitative feature can be represented as

$$
\left\{\begin{array}{c}
0,0 \leq x \leq \sum_{i=1}^{l-1} a_{i}-\frac{1}{2} \min _{a}\left(a_{l-2}, a_{l-1}, a_{l}\right) \\
1+\frac{x-\left(\sum_{i=1}^{l-1} a_{i}-\frac{1}{2} \min _{a}\left(a_{l-2}, a_{l-1}, a_{l}\right)\right)}{\min \left(a_{l-2}, a_{l-1}, a_{l}\right)}, \\
\sum_{i=1}^{l-1} a_{i}-\frac{1}{2} \min _{a}\left(a_{l-2}, a_{l-1}, a_{l}\right)<x \\
\leq \sum_{i=1}^{l-1} a_{i}+\frac{1}{2} \min _{a}\left(a_{l-2}, a_{l-1}, a_{l}\right) \\
\leq \sum_{i=1}^{l-1} a_{i}-\frac{1}{2} \min _{i}\left(a_{l-2}, a_{l-1}, a_{l}\right)<x \leq \\
1+\frac{x-\left(\sum_{i=1}^{l} a_{i}-\frac{1}{2} \min _{a}\left(a_{l-1}, a_{l}, a_{l+1}\right)\right)}{\min _{a}\left(a_{l-1}, a_{l}, a_{l+1}\right)}, \\
\sum_{i=1}^{l} a_{i}-\frac{1}{2} \min _{a}\left(a_{l-1}, a_{l}, a_{l+1}\right)<x \leq \\
\leq \sum_{i=1}^{l} a_{i}+\frac{1}{2} \min _{a}\left(a_{l-1}, a_{l}, a_{l+1}\right)<x \leq 1 \\
\leq a_{i}+\frac{1}{2} \min _{a}\left(a_{l-1}, a_{l}, a_{l+1}\right)
\end{array}\right.
$$

Based on the above, the subject area can be represented in the form of a multi-level environment consisting of many elements of the subject area, a variety of functions and methods working on these elements and a variety of properties of elements and relationships between elements, i.e., in the form of an ontology that includes description of the properties of the domain and the interaction of objects in some formal language that has logical semantics. If the system is complex and the number of factors is large, then taking into account all its characteristics (components) leads to extreme complexity. Therefore, only a limited number has to be entered into the model, and the remaining components must be taken into account without explicitly entering into the model, but taking into account their influence as a fuzzy reaction of the model to one or another choice of an alternative. Obviously, algebraic comparison of components is impossible and can be performed using fuzzy logic methods.

Based on the proposed method of forming the structure of the information system in the form of a set of interacting semantically defined and formalized objects connected with each other by hierarchical relations of the classes of attributes of the business processes that define them, it is most expedient to use as measurements when building a cube of sets obtained according to the rules of overlapping interacting accounting objects. The fact table in this case will contain integer columns giving the numerical characteristics of each dimension defined in this way, and several integer columns keys for accessing the dimension tables that decrypt them.

For each dimension, we compile a list of unique values from the elements stored in the columns and perform preliminary aggregation of facts for records that have the same dimension values. Using intermediate tables (the so-called cross tables), you can link elements of different tables to each other, for which each record in the dimension tables is assigned a list, the elements of which will be the numbers of facts, during the formation of which these dimensions were used. For facts, respectively, for each record, we will assign the values of the coordinates along which it is located in the hypercube. Dimensions have a hierarchical structure, consisting of one or more levels, on the basis of which the operations of the convolution or drill down are carried out.

For each free business process, a separate OLAP table can be created, the logical connection of which and the construction of OLAP tables of the next level is possible only at the level of aggregated analytical indicators reduced to a comparable form. In this case, we are talking about the transition to the management of data that are distributed across many repositories while providing a basic set of functions over all data sources - that is, to the management of data spaces. In some cases, the boundaries between data spaces can be floating, so all kinds of connections between participants must be formalized, and semantic integration develops in time only where required. This ensures a high level of component autonomy.

As a result, we can talk about operations on finite-dimensional vector spaces, represented by multidimensional cubes, where measurement vectors are used as bases. The composition of such cubes can be represented as a linear combination of basis vectors - a tensor product, the result of which is the most general space into which the original spaces can be displayed bilinear.

Consider the option of constructing the dimensions of a hypercube, for which we will make the necessary transformations of the data stored in the database tables. So, in order to improve performance when building a hypercube, we will find unique elements stored in columns that will be dimensions of the hypercube. For records with the same dimension values, let's perform preliminary aggregation. As mentioned above, the unique values in the measurement fields are important for us. To build slices of a hypercube, we need the following capabilities - defining coordinates (actually, measurement values) for table records, as well as defining records that have specific values. Actions are divided into two stages - according to the above classification of attributes. For formalized attributes, you can immediately create a table of unique values, on the basis of which the dimension will be built. Depending on the type of business processes to be integrated, we define the integration rule. We define keywords (phrases), compile a table of a set of options for 
their combinations that satisfy the semantic rule (by the membership function), and, as in the case of formalized attributes, build a table of unique values (Fig. 8). That is, instead of one table, we got an analogue of a normalized database. We are only interested in the coordinates in our hypercube, so we define the coordinates for the measurement values. The simplest thing is to renumber the values of the elements. In order for the numbering to be unambiguous within one dimension, let us first sort the lists of measurement values (dictionaries, in terms of a database) in alphabetical order.

\section{RESULTS AND DISCUSSION}

In practice, this concept finds its application in a Web-based environment, which requires high performance when processing large amounts of data, for example, a Web portal (department), which, in turn, is a set of database information transmission channels connected into a single system data, information storages, knowledge bases, as well as information technologies that support the processing, analysis and transfer of information at various levels of integration. Fig. 1 shows OLAP cube formation model.

At the same time, when solving problems of one system, knowledge of other systems is used. Similarly, the same objects in different systems can be described by different properties and, accordingly, have a different structure. One of the challenges is determining how to integrate the knowledge of different topics within a single Web portal. The main operation for a larger number of users is the operation of searching and obtaining information. Moreover, the data itself, once formed, is no longer subject to modification. Dynamic databases in such a situation are ineffective, and in the world practice today, to solve such problems, a transition to information storages is carried out. Let $D_{j}$ be a collection of documents, $M_{j}$ a set of queries, $d_{k}: M_{j} \rightarrow 2^{D_{j}}$ be a mapping that associates a set of documents with each query. Each information subsystem of the portal can be represented by a tuple $S_{j}=\left(D_{j}, M_{j}, d_{j}\right)$, where $j=1, \ldots, m-$ information subsystems used in the portal. Then the information system of the portal can be represented in the form of a distributed information system based on the global thesaurus $T$ and defined by the tuple $S=(T, D, M, d)$ through the local components $D=\mathrm{U}_{j} D_{j}$ and $d=\mathrm{U}_{j} d_{j}$. Any database can be viewed as an object archive designed to store attributes: passive objects that do not have state models, active objects subject to the life cycle. Fig. 2 shows the process of creating new OLAP cube based on the subject areas relations.

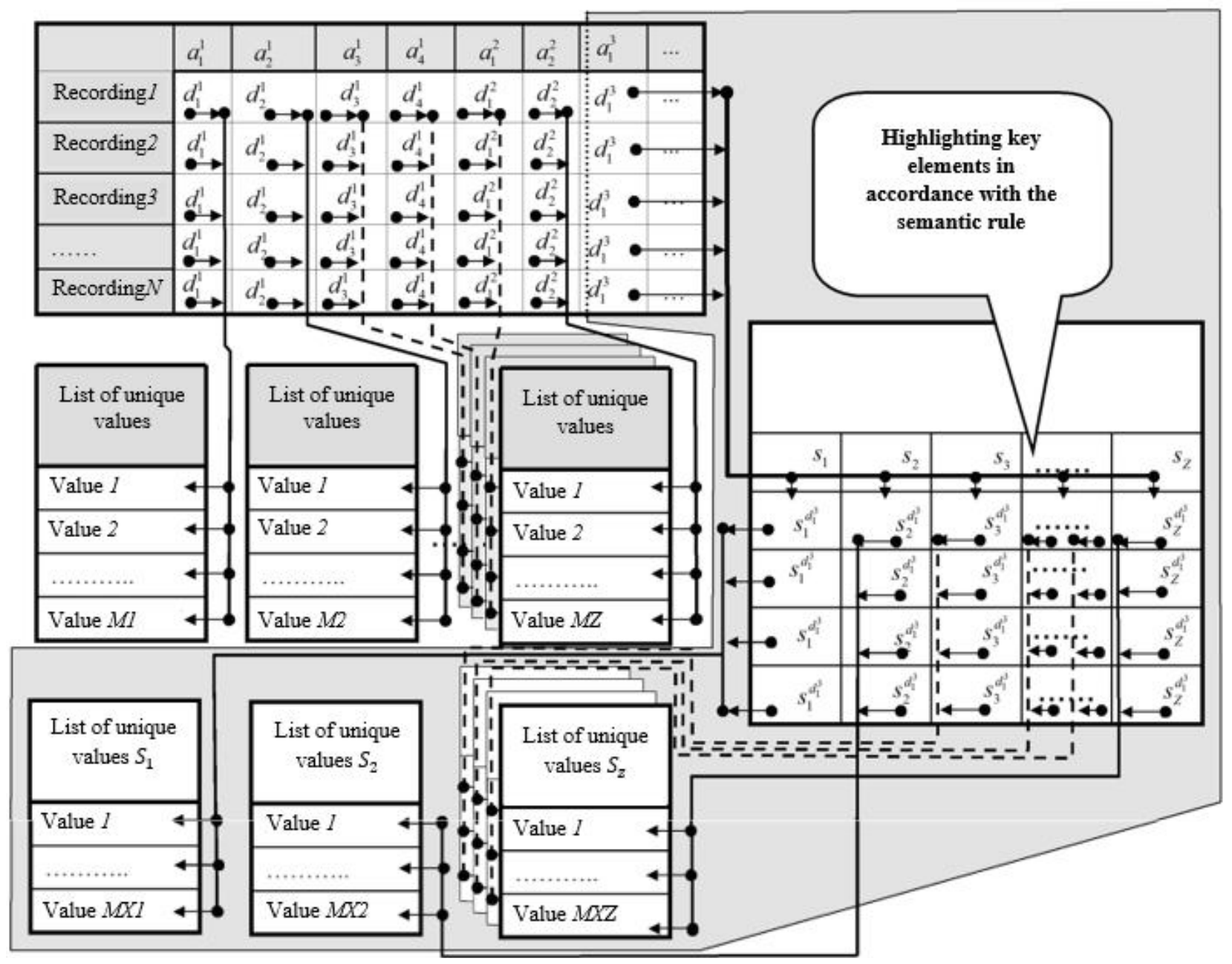

Figure 1: OLAP cube formation model 


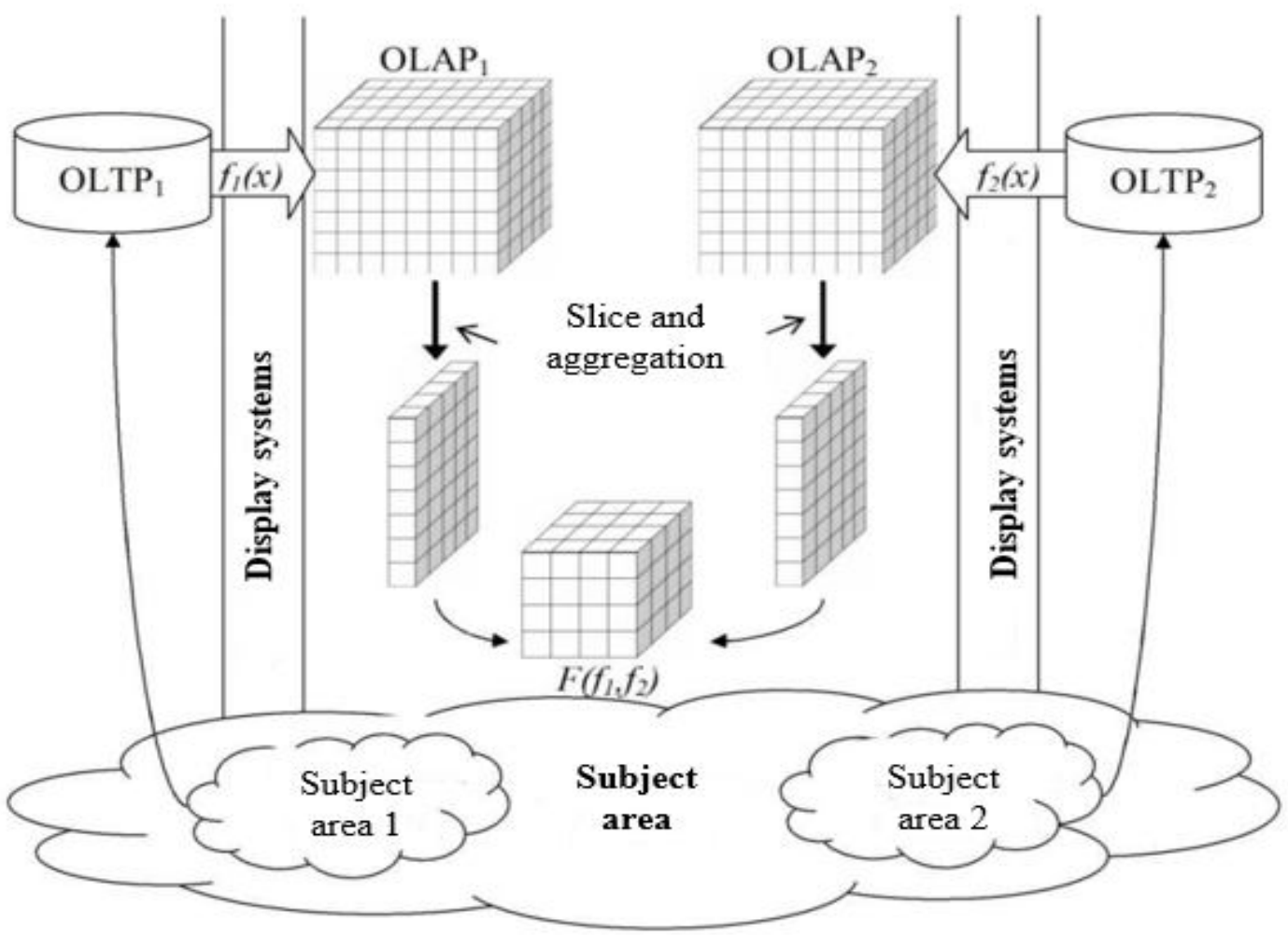

Figure 2: The process of creating a new OLAP cube based on the subject areas relations

There is a multi-tempo change in attributes. For example, the identity of a student does not change, and a number of specific attributes, such as faculty, group, current performance, are dynamic and modified. At the end of training, the data becomes permanent and can never be changed.

Thus, it is advisable to divide all the considered information into different levels, both in terms of lifetime and access, and the development of the database consists in adding and removing tuples corresponding to object instances. In this case, the database automatically goes into the category of information storages completely when the following conditions are met: the frequency of deleting tuples from the storage is commensurate with the time of its existence, and the OLAP cube is supplemented with a new dimension fragmented time. This is the moment of transferring information from the database to the storage, in accordance with the changed value of the attributes.

If the information in dynamic databases after modification is not of interest from any of the points of view, then the organization of an information storage based on such databases does not make sense. The OLAP cubes themselves, corresponding to OLTP systems, can be represented as multidimensional information objects with corresponding properties.

Since each OLTP system, being an implementation of a domain model, is mapped to a multidimensional matrix (OLAP cube), there are functions for this mapping. Considering the subject areas as part of the extended subject area, we can conclude that it is possible to establish a relation function between display functions, and, as a consequence, build a new system or OLAP cube based on this relation. It should be noted that the efficiency of the Web portal can be significantly improved if the features of the measurement scales are more accurately taken into account in modeling.

\section{CONCLUSION}

This article is devoted to the problem of the ability to represent business processes of complex systems of project and production management as a set of interacting semantically defined objects. To solve this problem, multidimensional modeling based on OLAP technology is proposed. The model covering the information system is presented in the form of a metabase, which contains information about each type of instance of an independent business process. A mathematical description of the model of the subject area from the standpoint of OLAP-tools is given. It is proposed to use a multidimensional data model or a hypercube, the edges of which are sequences of values of the analyzed parameters for multidimensional analysis of the subject area. Each OLTP system is an implementation of the subject area model and is mapped to a multidimensional matrix as OLAP-cube. The application of this concept in a Web-based environment is covered.

\section{REFERENCES}

1. P Kulikov, M. Dyomin, D. Chernyshev, D. Kuśnierz-Krupa and M. Krupa. The issues of preservation and revitalization of residential, public and industrial buildings from the second half of the 
19th and early 20th centuries in Kyiv and Krakow, Wiadomosci Konserwatorskie, Vol. 2019, Issue 60, 2019, pp. 140-146.

2. D. Chernyshev, D. Ryzhakov, O. Dikiy, O. Khomenko and S.Petrukha. Innovative Technology for Management Tools of Commercial Real Estate in Construction, International Journal on Emerging Trends in Engineering Research, 8(9), September 2020, pp. $\quad$ 4967-4973, https://doi.org/10.30534/ijeter/2020/13892020

3. J. Marchuk, D Ryzhakov, G. Ryzhakova, S. Stetsenko. Identification of the basic elements of the innovation-analytical platform for energy efficiency in project financing, Investment Management and Financial Innovations, Volume 14, Issue 4, 2017, pp. 12-20, http://dx.doi.org/10.21511/imfi.14(4).2017.02

4. T. Honcharenko, Y. Chupryna, I. Ivakhnenko, M. Zinchenco, T. Tsyfra. Reengineering of the Construction Companies Based on BIM-technology, International Journal of Emerging Trends in Engineering Research, 8(8), August 2020, pp. 4166-4172 https://doi.org/10.30534/ijeter/2020/22882020

5. D. Ryzhakov, O. Dikiy, M. Druzhynin, H. Petrenko and T. Savchuk. Innovative tools for management the lifecycle of strategic objectives of the enterprise-stakeholder in construction, International Journal on Emerging Trends in Engineering Research, $8(8), \quad 2020, \quad$ pp. 4526-4532, https://doi.org/10.30534/ijeter/2020/78882020

6. Y. Ivashko, D. Chernyshev and P. Chang. Functional and figurative and compositional features of traditional Chinese pavilions, Wiadomosci Konserwatorskie, Vol. 2020, Issue 61, 2020, pp. 60-66.

7. M. Dyomin, A. Dmytrenko, D. Chernyshev and Y. Ivashko. Big Cities Industrial Territories Revitalization Problems and Ways of Their Solution, Lecture Notes in Civil Engineering, Vol.73, 2020, pp. 365-373.

8. V. Mihaylenko, T. Honcharenko, K. Chupryna, Yu. Andrashko and S. Budnik. Modeling of Spatial Data on the Construction Site Based on Multidimensional Information Objects. International Journal of Engineering and Advanced Technology. Volume-8 Issue-6, pp.3934-3940, August 2019. URL: https://www.ijeat.org/wp-content/uploads/papers/v8i6/F 9057088619.pdf

9. I. Arutiunian, M. Poltavets, O. Bondar, V. Anin, F. Pavlov. Structural Information Management of Production Systems in Construction, International Journal of Advanced Trends in Computer Science and Engineering, 9(4), July -August 2020, pp. 4794 - 4797, https://doi.org/10.30534/ijatcse/2020/87942020

10. A.V. Burkov, R.V. Pshenichnov, T.V. Yalyalieva. Construction of models for conversion of mortgage applications by the method of multiple regression and Neural Networks, International Journal of Advanced Trends in Computer Science and Engineering, 9(4), July -August 2020, pp. 4546-4550, https://doi.org/10.30534/ijatcse/2020/52942020
11. K.I. Kyivska, S.V.Tsiutsiura, M.I.Tsiutsiura, O.V.Kryvoruchko, A.V.Yerukaiev and V.V.Hots. A study of the concept of parametric modeling of construction objects, International Journal of Advanced Research in Engineering and Technology, Volume 10, Issue 2, 2019, pp 636-646.

12. Y. Riabchun, T. Honcharenko, V. Honta, K.Chupryna and O.Fedusenko. Methods and means of evaluation and development for prospective students' spatial awareness, International Journal of Innovative Technology and Exploring Engineering, Volume 8, Issue 11, September 2019, pp. 4050-4058, https://www.ijitee.org/wp-content/uploads/papers/v8i11/ K15320981119.pdf

13. O. Shushura, L. Asieieva, I. Husyeva, M. Stepanov, O.Datsiuk. Construction of models for conversion of mortgage applications by the method of multiple regression and Neural Networks, International Journal of Advanced Trends in Computer Science and Engineering, 9(3), May-June 2020, pp. 2702-2707, https://doi.org/10.30534/ijatcse/2020/33932020

14. A. G. Myasnikov. Information and logical modeling in construction, International Journal of Advanced Trends in Computer Science and Engineering, 9(1), January -February 2020, pp. 304-307, https://doi.org/10.30534/ijatcse/2020/46912020

15. O. Terentyev, S. Tsiutsiura, T. Honcharenko and T. Lyashchenko. Multidimensional Space Structure for Adaptable Data Model, International Journal of Recent Technology and Engineering (IJRTE), Volume-8 Issue-3, pp. 7753-7758, September 2019. URL: https://www.ijrte.org/wp-content/uploads/papers/v8i3/C 6318098319.pdf

16. M. Ahmad, T. Sinelnikova, S. Mustafa, V. Lyashenko. Features of the Construction and Control of the Navigation System of a Mobile Robot. International Journal on Emerging Trends in Engineering Research, $8(4), \quad$ pp. $\quad 1445-1449, \quad$ April 2020. https://doi.org/10.30534/ijeter/2020/82842020

17. S. A. De Santana. Modeling urban landscape: new paradigms and challenges in territorial representation, Disegnare con., 2013, no. 6(11), pp. 161-174, DOI 10.6092/issn.1828-5961/3379

18. A. Kuchansky, Y. Andrashko, A. Biloshchytskyi, O. Danchenko, O. Ilarionov, I. Vatskel and T. Honcharenko. The method for evaluation of educational environment subjects' performance based on the calculation of volumes of $M$-simplexes in Eastern-European Journal of Enterprise Technologies, 2 (4-92), pp. 15-25, http://journals.uran.ua/eejet/article/download/126287/12 5235

19. A. Kuchansky, A. Biloshchytskyi, Yu.Andrashko, S. Biloshchytska, T. Honcharenko, V.Nikolenko. Fractal Time Series Analysis in Non-Stationary Environment, 2019 IEEE International Scientific-Practical Conference: Problems of Infocommunications Science and Technology, PIC S and T 2019 - Proceedings, 2019, pp. 236-240. 